\title{
Mesenteric Adenitis Caused by Salmonella Typhi: A Case Report
}

\author{
Nur Ain C. A ${ }^{a}$, Nik Haszroel Hysham N. H. ${ }^{a, b}$ \\ ${ }^{a}$ Department of Medical Microbiology and Parasitology, School of Medical Sciences, Universiti Sains Malaysia, Health Campus, Kelantan, Malaysia \\ ${ }^{\mathrm{b}}$ Hospital Universiti Sains Malaysia, Universiti Sains Malaysia, Health Campus, Kubang Kerian, Kelantan, Malaysia
}

\section{ABSTRACT}

Both mesenteric adenitis and acute appendicitis may present with fever and right lower quadrant pain. This presentation makes it challenging to provide a timely and accurate diagnosis to avoid unnecessary surgery. We report a case of an 8-year-old who was admitted with fever and right lower quadrant pain, loss of appetite, and diarrhoea. There were tenderness and rigidity in the right lower quadrant of the abdomen. Hepatomegaly was present. Full blood count showed pancytopenia, and serum transaminases were mildly elevated. The clinical features in typhoid fever are not specific, making it difficult to differentiate from other infectious causes such as malaria, dengue, or leptospirosis, common in the region. This report illustrates a rare case of mesenteric adenitis caused by Salmonella serotype Typhi. It also shows the benefit of doing an abdominal ultrasound to help the surgeon decide whether surgery is necessary.

KEYWORDS: Mesenteric adenitis, Typhoid fever, Salmonella Typhi, acute appendicitis

\section{INTRODUCTION}

Mesenteric adenitis and acute appendicitis both may present with fever and right lower quadrant pain. This presentation makes it challenging to provide a timely and accurate diagnosis to avoid unnecessary surgery. Most cases of mesenteric adenitis are caused by Yersinia enterocolitica and Yersinia pseudotuberculosis. The clinical features in typhoid fever are not specific, making it difficult to differentiate from other infectious causes such as malaria, dengue, or leptospirosis, common in the region. We found that there are limited case reports on mesenteric adenitis caused by Salmonella Typhi infection. ${ }^{1}$

\section{CASE REPORT}

An 8-year-old, with no underlying disease, was admitted with one week of fever and two days of colicky right

\section{Corresponding Author:}

Dr. Nik Haszroel Hysham Nik Hashim

Department of Medical Microbiology and Parasitology,

School of Medical Sciences, Universiti Sains Malaysia,

Health Campus, 16150 Kubang Kerian,

Kelantan, Malaysia.

Tel No : +609-767 6246

E-mail : haszroel@usm.my lower quadrant abdominal pain, loss of appetite, and diarrhoea. On admission, she was febrile with a temperature of $39^{\circ} \mathrm{C}$. Her blood pressure and pulse rate were normal at 105/50 $\mathrm{mmHg}$ and 115 beats per minute, respectively. There were tenderness and rigidity in the right lower quadrant of the abdomen. The liver was palpable three fingerbreadths below the subcostal margin. However, the spleen was not palpable. She had mild anaemia with a haemoglobin level of $9.8 \mathrm{~g} / \mathrm{dL}$, leukopenia with a total white blood cell count of $2.87 \mathrm{x}$ $10^{3} \mu \mathrm{L}$, and thrombocytopenia with a platelet count of $113 \times 10^{3} \mu \mathrm{L}$. Her C-reactive protein was high at $78 \mathrm{mg} /$ dL. Her liver function test showed transaminitis with aspartate aminotransferase (AST) level of $114 \mathrm{U} / \mathrm{L}$ and alanine aminotransferase (ALT) level of $89 \mathrm{U} / \mathrm{L}$. The working diagnosis on admission was an acute abdomen with pancytopenia and transaminitis. The differential diagnosis was leptospirosis, dengue fever, and typhoid fever.

Leptospirosis $\operatorname{IgM}$ and Dengue NS1 antigen, IgG, and IgM were all negative. Typhoid serology by immunochromatography assay (ICT) was positive for Salmonella Typhi IgM and negative for IgG. No 
abnormality was seen on abdominal X-ray. Abdominal ultrasonography showed that there was no sonographic evidence of acute appendicitis. The liver was enlarged $13.6 \mathrm{~cm}$ in the right midclavicular line with moderate ascites and multiple reactive mesenteric lymphadenitis seen. The largest lymph node was 1.7 x $0.8 \mathrm{~cm}$ in size. Blood culture was positive for Salmonella enterica serotype Typhi and was found to be susceptible to ampicillin, ceftriaxone, and ciprofloxacin. However, the stool culture result was negative. The final diagnosis was revised to typhoid fever with mesenteric adenitis and transaminitis. She was put on intravenous ceftriaxone $1 \mathrm{~g}$ every 12 hours $(50 \mathrm{mg} / \mathrm{kg} /$ dose; her body weight was 22 $\mathrm{kg}$ ) for one week. She showed marked improvement with the treatment given. The abdominal pain resolved, became afebrile, and blood parameters improved. She was discharged from the ward with oral ciprofloxacin for another week.

\section{DISCUSSION}

Mesenteric adenitis is a condition characterized by inflammation in the mesenteric lymph nodes. It can be primary or secondary to various infective, malignant or inflammatory disorders. ${ }^{2}$ Both acute appendicitis and mesenteric adenitis typically present with fever and right lower quadrant pain. Clinically, both are almost indistinguishable. However, acute appendicitis is a possibly life-threatening condition and often requires urgent surgery. Mesenteric adenitis is usually selflimiting and can be managed conservatively. A lot of unnecessary surgery can be avoided if we can differentiate the two. An essential distinguishing sign is rebound tenderness, which is often present in acute appendicitis but not in mesenteric adenitis. ${ }^{3}$ Abdominal ultrasound is a vital tool to help differentiate between the two. ${ }^{4}$

Mesenteric adenitis caused by specific organisms needs to be treated with antimicrobials specific to it. Though various organisms can cause mesenteric adenitis, the most reported is Yersinia enterocolitica and Yersinia pseudotuberculosis. ${ }^{5}$ However, this patient was infected by Salmonella serotype Typhi.

Clinical diagnosis of typhoid fever is not easy as the clinical manifestation of the disease varies greatly depending on various factors, and the patient population studied. The major differential diagnoses for fever and abdominal pain include typhoid fever, malaria, dengue, and leptospirosis. Both typhoid fever and malaria may produce fever and hepatosplenomegaly. In typhoid fever, the fever is usually described as low-grade in the first week, which gradually increases in a stepladder pattern and becomes continuous high-grade in the second week. In comparison, the fever in malaria usually has an intermittent pattern. $^{6}$ In contrast, the clinical presentation in dengue is usually petechiae rash and thrombocytopenia. According to a study, thrombocytopenia can occur in $10-15 \%$ of children with typhoid fever. ${ }^{7}$

In typhoid fever, Salmonella Typhi colonizes the bone marrow, liver, and spleen. The presence of mild pancytopenia and a mild elevation in the serum transaminases but normal alkaline phosphatase supports this diagnosis. ${ }^{6,8}$ In contrast, leukocytosis is expected in acute appendicitis and lymphocytosis in mesenteric adenitis. $^{2}$

Malaria can be diagnosed relatively quickly with a blood smear to look for malarial parasites. However, serological diagnosis of typhoid fever, such as the Widal test, can produce false-positive results due to cross-reaction with other infections. ${ }^{8}$ The definitive diagnosis of typhoid fever requires Salmonella serotype Typhi's isolation from blood, stool, urine, or bone marrow, which takes some time to obtain. ${ }^{9}$ The sensitivity of blood culture is only 40-60\%. ${ }^{10}$ Prior antimicrobial usage before blood sampling can lead to false-negative results. In this patient, the antibiotic was started when her typhoid serology by immunochromatography assay (ICT) was positive for Salmonella Typhi IgM and negative for IgG. The blood culture result later confirmed the cause of the infection.

\section{CONCLUSION}

Both mesenteric adenitis and acute appendicitis may present with fever and right lower quadrant pain. This presentation makes it challenging to provide a timely and accurate diagnosis to avoid unnecessary surgery. This report illustrates a rare case of mesenteric adenitis caused by Salmonella Typhi. It also shows the benefit of doing an 
abdominal ultrasound to help the surgeon decide whether surgery is necessary.

\section{REFERENCE}

1. Likitnukul S, Wongsawat J, Nunthapisud P. Appendicitis-like syndrome owing to mesenteric adenitis caused by Salmonella typhi. Ann Trop Paediatr 2002; 22(1):97-9.

2. Gross I, Siedner-Weintraub Y, Stibbe S, Rekhtman D, Weiss D, Simanovsky N, et al. Characteristics of mesenteric lymphadenitis in comparison with those of acute appendicitis in children. Eur J Pediatr 2017; 176(2):199-205.

3. Lee JH, Rhee PL, Lee JK, Lee KT, Son HJ, Kim JJ, et al. The etiology and clinical characteristics of mesenteric adenitis in Korean adults. J Korean Med Sci 1997; 12(2):105-10.

4. Toorenvliet B, Vellekoop A, Bakker R, Wiersma F, Mertens B, Merkus J, et al. Clinical differentiation between acute appendicitis and acute mesenteric lymphadenitis in children. Eur J Pediatr Surg 2011; 21(2):120-3.

5. Blattner RJ. Acute mesenteric lymphadenitis. The Journal of Pediatrics 1969; 74:479-81.

6. Cunha BA. Typhoid fever: the temporal relations of key clinical diagnostic points. Lancet Infectious Disease 2006; 6:318-20.

7. Margaret AP, Solomon P, Lohith H. A rare case of typhoid presenting with fever, ascites, hyponatremia, thrombocytopenia, mesenteric lymphadenitis, and multi-drug resistance. J Pharm Bioallied Sci 2015; 7 (Suppl 1):S65-6.

8. Connor BA, Schwartz E. Typhoid and paratyphoid fever in travellers. Lancet Infectious Diseases 2005; 5(10):623-8.

9. Bhan MK, Bahl R, Bhatnagar S. Typhoid and paratyphoid fever.The Lancet 2005; 366 (9487):749_ 62.

10. Bhutta ZA. Current concepts in the diagnosis and treatment of typhoid fever. British Medical Journal 2006; (333)78-82. 